\title{
Dark Matter Searches at LNF
}

\author{
Claudio Gatti ${ }^{1}$, Paola Gianotti ${ }^{1}{ }^{*}$, Carlo Ligi ${ }^{1}$, Mauro Raggi ${ }^{2}$ and Paolo Valente ${ }^{3}$ \\ 1 Laboratori Nazionali di Frascati dell'INFN, Via E. Fermi 54, 00044 Frascati, Italy; \\ claudio.gatti@lnf.infn.it (C.G.); carlo.ligi@lnf.infn.it (C.L.) \\ 2 Department of Physics, Sapienza Università di Roma p.le A. Moro 5, 00185 Roma, Italy; \\ mauro.raggi@uniroma1.it \\ 3 INFN, Sezione di Roma p.le A. Moro 5, 00185 Roma, Italy; paolo.valente@roma1.infn.it \\ * Correspondence: paola.gianotti@lnf.infn.it
}

check for updates

Citation: Gatti, C.; Gianotti, P.; Ligi, C.; Raggi, M.; Valente, P. Dark Matter Searches at LNF. Universe 2021, 7, 236. https: / / doi.org/10.3390/ universe7070236

Academic Editors: Alexander

Andrianov, Marco Selvi and

Francesco Terranova

Received: 31 May 2021

Accepted: 6 July 2021

Published: 9 July 2021

Publisher's Note: MDPI stays neutral with regard to jurisdictional claims in published maps and institutional affiliations.

Copyright: (c) 2021 by the authors. Licensee MDPI, Basel, Switzerland. This article is an open access article distributed under the terms and conditions of the Creative Commons Attribution (CC BY) license (https:// creativecommons.org/licenses/by/ $4.0 /)$.

\begin{abstract}
In recent years, the absence of experimental evidence for searches dedicated to dark matter has triggered the development of new ideas on the nature of this entity, which manifests at the cosmological level. Some of these can be explored by small experiments with a short timescale and an investment that can be afforded by national laboratories, such as the Frascati one. This is the main reason why a laboratory that, traditionally, was focused in particle physics studies with accelerators has begun intense activity in this field of research.
\end{abstract}

Keywords: research infrastructure; dark matter; axions; dark photons

\section{Introduction}

The Frascati National Laboratory of INFN has always been at the forefront of scientific research in the field of fundamental particle physics and in that of particle accelerator development. These two sectors have strongly overlapped since the first $\mathrm{e}^{+} \mathrm{e}^{-}$collisions, realized in ADA (the first world particle collider), as well as in more recent times with the still ongoing experimental activity at the DA $\Phi$ NE collider.

With the recent approval of the EuPRAXIA project [1,2], the laboratory is entering a new era. In less than ten years, a new machine will be built, with the ambitious goal of becoming the first user facility that exploits plasma acceleration. This machine will serve a vast community of users, which, however, will be outside of the fundamental physics sector. Therefore, to continue to play a role in the community of particle physics, a diversification of the scientific program of the laboratory is ongoing.

A critical analysis of the available infrastructures showed that some relevant measurements could be performed in the search for extensions of the Standard Model to explain the nature of dark matter. The theoretical background of this program has its roots in the hypothesis that dark matter could be explained by admitting low energy feebly interacting particles as an alternative to the WIMPs paradigm.

This paper illustrates the present and the near future programs of the laboratory in the sector of dark matter search. After an overview of the available infrastructures, the details of the two pillars that constituted this program are given: the search for QCD axions and the investigation of the existence of dark sectors .

\section{The Frascati National Laboratory}

The Frascati National Laboratory (LNF) is the largest and the first built of the four laboratories that the Italian Institute for Nuclear Physics (INFN) owns. The Institute was founded almost seventy years ago with the primary intention to provide Italy with its first particle accelerator: an electron-synchrotron of $1.1 \mathrm{GeV}$ energy. Since then, the research activities carried out at LNF have always been in the fields of high energy physics, accelerator operation and development, and interdisciplinary research, with a perfect 
balance between internal activities, taking place on site, and external ones conducted in the major laboratories all over the world.

The LNF is also the place where the first matter-antimatter collider was built: AdAan electron-positron collider with a center of mass energy of $500 \mathrm{MeV}$ [3]. AdA was not conceived as an accelerator to provide beam for experiments, but it was an experiment itself. The goal was to demonstrate that particles and antiparticles could be accelerated and then collide, in proper points, in the same vacuum pipe. After the success of this pioneering enterprise, the largest $\mathrm{e}^{+} \mathrm{e}^{-}$collider was built: ADONE, with a center of mass energy of $3 \mathrm{GeV}$ [4]. ADONE's experiments revolved around QED tests, proton and neutron form factors, muon study, and multi-hadron production.

ADONE operated at LNF from 1969 to 1993, when it was dismounted to leave the floor to a collider of new conception: DA $\Phi N E$ [5]. DA $\Phi N E$ is a $\Phi$-factory, i.e., a collider whose c.m. energy was selected to produce $\phi$ mesons at a high rate. The experiments conducted at DA $\Phi N E$ concerned precision studies of CP-violation (KLOE) [6], low energy QCD (KLOE2 [7], DEAR [8], SIDDHARTA [9]), and hypernuclear physics (FINUDA) [10].

Today, LNF stands over an area of 135,000 $\mathrm{m}^{2}$, of which 56,000 are indoors and which includes offices, laboratories, and service areas. For what concerns the accelerator infrastructures, this is what is available at LNF:

- DA $\Phi \mathrm{NE}$, an $\mathrm{e}^{+} \mathrm{e}^{-}$collider, unique in Europe, operated at $\phi$ energy and able to deliver instantaneous luminosities $\mathcal{L} \sim 2 \times 10^{32} \mathrm{~cm}^{-2} \mathrm{~s}^{-1}$.

- DA $\Phi N E-l i g h t$, a synchrotron light laboratory, housing several synchrotron radiation lines extracted from the electron ring of DA $\Phi N E$ in the soft- $X$ and infrared range.

- a Beam Test Facility, BTF, an experimental area equipped for detector and beam diagnostic tests. Here, two beam lines, extracted from the DAФNE LINAC, can provide beams of electrons, positrons, photons of variable intensity and energy [11].

- SPARC_LAB, a complex hosting a photo-injector that can produce high brightness electron beams up to $170 \mathrm{MeV}$ (SPARC), which feeds a 12-m long undulator for FEL generation and a laser (FLAME) of power $\sim 200$ TW. The SPARC_LAB is an infrastructure for R\&D on new techniques of particle acceleration and for interdisciplinary studies, including PWFA and LWFA experiments, TeraHertz radiation and a Compton source [12].

Figure 1 shows an aerial view of the LNF premises with the main infrastructures previously mentioned indicated.

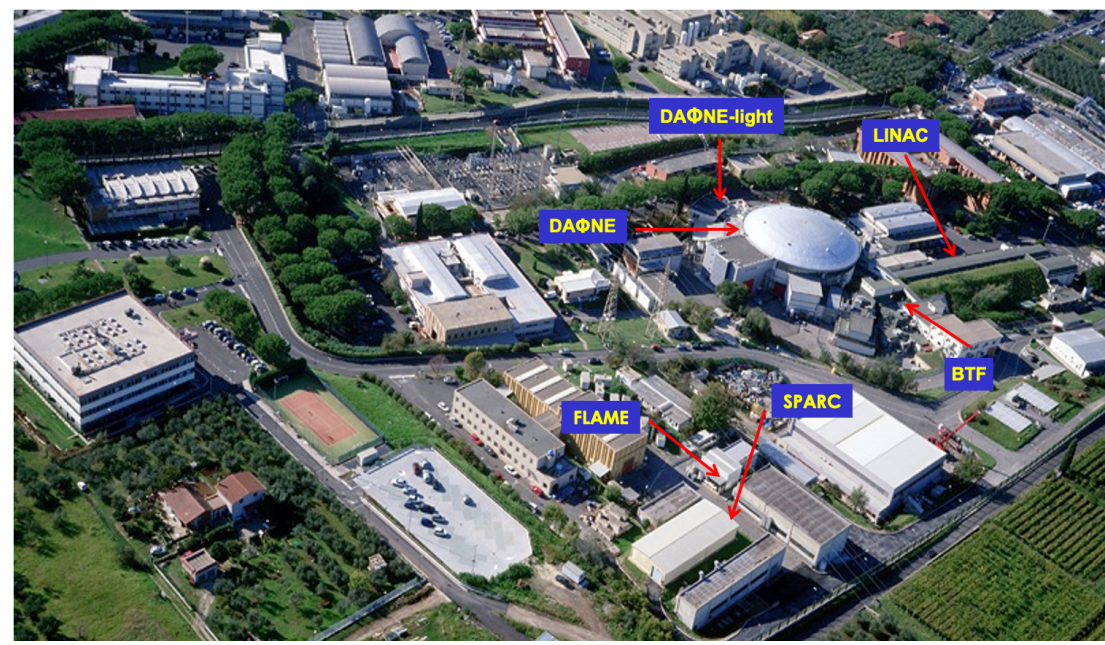

Figure 1. Aerial view of the Laboratori Nazionali di Frascati (LNF); the main infrastructures of the laboratory are indicated (see text for more details).

Since the early days, together with the development, construction, and operation of accelerators, LNF activity has been strongly committed to the design and construction of 
the forefront of detectors for particle, nuclear, and astroparticle physics. LNF hosts large assembly halls with several clean rooms (for a total surface of about $480 \mathrm{~m}^{2}$ ) equipped with special tools for designing and building large and complex experimental equipment.

These are continuously used to contribute to experiments taking place in the major laboratories all over the world. At present, for instance, some of the upgrades of the LHC apparata (i.e., ATLAS, CMS, LHCb, and ALICE) are taking place in these halls. To complete the panorama a mechanical workshop, an electronic service, a powerful and modern computing center, involved in the GRID Tier2 network, and a medical physics service unique within INFN are also available .

As already mentioned, the research activity ongoing at LNF comprises on-site and external projects. In recent years, the center of the on-site work has been the DA $\Phi$ NE complex; however, this era, after about twenty years of operation, is coming to an end. As a general consideration, forefront particle physics has become something that, due to the costs and dimensions, cannot be afforded by national laboratories. On the other side, to justify big infrastructures and resources, a laboratory should have the capability to produce in-house scientific results. This is the main reason that triggered, at LNF, a widening of interests and started the development of new activities in the field of the dark matter. In the following sections, all the details of this research program are discussed.

\section{The Problem of Dark Matter}

The idea of the existence in the universe of some form of non-conventional matter dates back to cosmological studies carried out in the first half of twentieth century. Currently, the observational evidence of some form of matter leftover from the Big Bang that produces gravitational effects but is almost not subject to electromagnetic interaction, generically referred to as "cold dark matter" (DM), increased and this concept then became part of modern cosmological models [13].

By using different approaches (i.e., mass-to-light ratio calculations, stellar velocity determination, and the virial theorem) cosmologists computed an amount of DM in the universe in about $84.4 \%$ of the total matter density. However, admitting its existence does not imply that its nature is known. At present, the most accredited scenario considers DM constituted by new particles almost electrically neutral, cold, collisionless, or selfinteracting [14]. Few constraints about the mass are possible, and model predictions span a very wide range (i.e., $10^{-22} \div 10^{12} \mathrm{eV}$ [15]), while, for the lifetime, this should be of the order of the age of the universe.

The Standard Model (SM) of particle physics does not offer any candidate fitting these requirements, and this, together with the need to explain some observed anomaly, has triggered the development of various extensions of the SM that could also account for the DM. Examples of the new elaborated theories include weakly-interacting massive particles (WIMPs) motivated by the weak hierarchy problem, such as neutralinos in the supersymmetric models [16], as well as extremely light axion particles [17] associated with the solution to the strong CP problem in QCD [18]. The cosmic abundance of these particles turned out to be close to the DM abundance inferred from the cosmological observations, and this coincidence has motivated decades of efforts of experimental searches (for a recent review of WIMP theory and experimental searches see [19]).

Given its importance, there is urgency in identifying the physics of dark matter, and this has made its search more broad. For example, there are no reasons why it cannot be produced at particle accelerators, and any running particle physics experiment looking for phenomena beyond the SM is implicitly also participating in this hunt.

On the other hand, the absence of sizable DM interactions with ordinary matter gives credit to the hypothesis that it consists of particles neutral under SM forces but perhaps charged under new forces not yet discovered. In general, they should be similar in structure to the known ones, but coupled only indirectly to ordinary matter. This scenario has been described by many theoretical extensions of the SM, and, in addition to gravity, there are few well-motivated interactions allowed by SM symmetries that provide a "portal" from the 
ordinary sector into the dark sector [20]. "While particle physics has traditionally focused on exploring matter at ever-smaller scales through high-energy experiments, testing this dark-sector hypothesis requires innovative low energy experiments that use high-intensity beams and/or high-sensitivity detectors [21]".

This novel approach to the DM problem gave impulse to a new set of dedicated experiments and/or of new analyses of existing data, together with an intense theoretical work. In this new landscape, the LNF decided to play a role, and two specific subjects were identified as accessible with the available know-how and the above mentioned infrastructures.

\section{Search for the QCD Axion}

The axion is an hypothetical particle introduced to solve the strong CP problem of QCD [18,22-24]. Provided their mass is in the range $1 \mu \mathrm{eV}<m_{a}<10 \mathrm{meV}$, axions can contribute significantly to the energy density of the universe in the form of DM clustered inside galactic halos $[17,25-27]$ and are possibly observable by means of detectors called haloscopes. A haloscope $[28,29]$ consists of a resonant cavity immersed in a static magnetic field that stimulates the axion conversion into photons through the Primakoff effect. When the cavity resonant frequency $v_{\mathrm{c}}$ is tuned to the axion mass $m_{a} c^{2} / h$, the expected power deposited by DM axions is given by [30]

$$
P_{a}=\left(\frac{g_{a \gamma \gamma}^{2}}{m_{a}^{2}} \hbar^{3} c^{3} \rho_{a}\right) \times\left(\frac{\beta}{1+\beta} \omega_{c} \frac{1}{\mu_{0}} B_{0}^{2} V C_{010} Q_{L}\right),
$$

where $\rho_{a} \sim 0.4-0.45 \mathrm{GeV} / \mathrm{cm}^{3}$ is the local DM density, and $g_{a \gamma \gamma}$ is the coupling constant appearing in the Lagrangian describing the axion-photon interaction. The second parenthesis contains the vacuum permeability $\mu_{0}$, the magnetic field strength $B_{0}$, the cavity volume $V$, its angular frequency $\omega_{c}=2 \pi v_{c}$, the coupling between cavity and receiver $\beta$, and the loaded quality factor $Q_{L}=Q_{0} /(1+\beta)$, with $Q_{0}$ as the unloaded quality factor. $C_{010}$ is a geometrical factor equal to about 0.69 for the TM010 mode of a cylindrical cavity. The typical signal power is on the order of $10^{-23} \mathrm{~W}$. Several experiments rely on the haloscope or similar concept: ADMX [31,32], HAYSTAC [33,34], ORGAN [35], CAPP [36,37], RADES [38], QUAX [30,39] and KLASH [40], MADMAX [41], BRASS [42], ABRACADABRA [43], and CASPER [44].

An important figure of merit of a haloscope is the time required to probe a region of axion masses with a given sensitivity, summarized by the scanning rate:

$$
\frac{d m_{a}}{d t} \propto \frac{B_{0}^{4} V^{2} Q_{L}}{T_{s y s}^{2}}
$$

where $T_{s y s}$ is the effective noise temperature of the system.

\subsection{The QUAX Experiment at LNF}

The QUAX (QUaerere AXion) experiment was proposed as a modified haloscope containing magnetized media to detect galactic axions through their coupling to the electron spin [45]. The first QUAX haloscope, at the Laboratori Nazionali di Legnaro (LNL) of INFN led to limits both for the axions coupling to electrons [39,46], the QUAXae experiment, and the coupling to photons [30,47], the QUAXa $\gamma$ experiment. With the latest results, the QUAX Collaboration reached a sensitivity to QCD axions in the mass region around $40 \mu \mathrm{eV}$. Further results were obtained in the development of both superconducting [48] and dielectric $[49,50]$ resonant cavities able to operate with high quality-factor in a strong magnetic field.

A second haloscope is under construction at the COLD Laboratory [51] at LNF. A dilution refrigerator was put in operation, a Leiden CF-CS110-1000, with a Sumitomo pulse-tube with 1.5-W cooling power at $4.2 \mathrm{~K}$, a base temperature of $8 \mathrm{mK}$, and a mixing 
chamber plate of $490 \mathrm{~mm}$ in diameter. The cooling power of the dilution refrigerator was measured to be $450 \mu \mathrm{W}$ at $100 \mathrm{mK}$ that will be extended up to $700 \mu \mathrm{W}$ by improving the pumping capacity with a new turbo pump.

The refrigerator is instrumented with four 0.86-mm BeCu-Ag-CuNi coaxial lines thermalized at different temperatures down to $4 \mathrm{~K}$ down to the mixing chamber plate. The signal is read out through a fifth dedicated superconducting coaxial line connecting a sample holder at $10 \mathrm{mK}$, designed to host an amplifier or a single photon-device, to the HEMT amplifier mounted on the 4-K plate and, with a normal line, up to an SMA feedthrough on a $300-\mathrm{K}$ flange and room temperature electronics.

The LNF haloscope will operate with a multicavity scheme, as proposed in [35], with the cavities tuned to different frequencies. Up to seven resonant cavities with the frequency between 8.5 and $10 \mathrm{GHz}$ may fit inside a newly purchased 9-T magnet with a $10-\mathrm{cm}$ bore taking into account the thickness of the cryostat radiation-shields and cavity walls. A successful operation of the cavities at the same frequency would further improve the scanning rate of the experiment. A realistic scan bandwidth of $\mathrm{O}(1) \mathrm{GHz}$ is feasible covering the yet unexplored region from about 9 to $10 \mathrm{GHz}$, while the haloscope at LNL would cover the region from 10 to $11 \mathrm{GHz}$, corresponding in total to the axion mass range from about 35 to $45 \mu \mathrm{eV}$.

Low-noise signal-amplification from an array of resonant cavities requires a large bandwidth quantum-limited amplifier. Within the project DART WARS (Detector Array Readout with Traveling Wave AmplifieRS) are under test Traveling Wave Josephson Parametric Amplifiers (TWJPA) chips fabricated at INRiM (right panel in Figure 2). TWJPAs are based on a coplanar waveguide composed of an array of RF-SQUIDs [52]. The non-linearity of the inductance associated to the Josephson junctions gives rise to a mixing process that is exploited to transfer energy from a pump signal to a signal tone.

The INRiM's implementation of this device, based on common Aluminum nanofabrication technology, consists of a repetition of 990 elementary cells with a periodicity of $63 \mu \mathrm{m}$ for a total length of about $6.25 \mathrm{~cm}$. The device can operate both in a three-wave and in a four-wave mixing regime with an expected amplification ranging from 25 to $35 \mathrm{~dB}$ in a bandwidth from 5 to $10 \mathrm{GHz}$ depending on the operation mode. TWJPAs are already used to readout arrays of qubits [53] coupled, through a readout cavity and a bandpass filter to a feedline terminated to a TWJPA for signal amplification in a 1.2-GHz wide band.

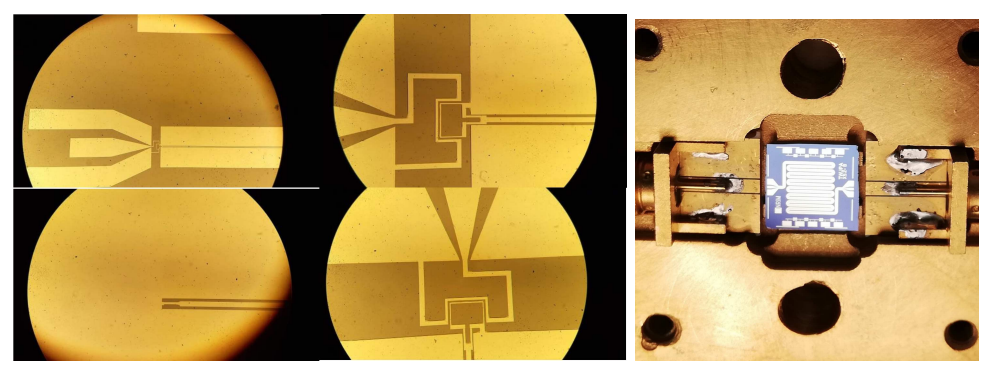

Figure 2. Left: optical image of the RF-chip with JJ and DC-SQUID fabricated at CNR-IFN for the SIMP project. Right: TWJPA from INRiM inside the LNF sample holder before bonding.

Resonant cavities will be fabricated using Type II superconductors allowing for improvement of the quality factor with respect to copper cavities even when operating in a magnetic field of several Tesla. Quality factors above 300,000 have been measured inside a 6-T magnetic field with a NbTi cavity $[47,48]$. An YBCO cavity was characterized at $7 \mathrm{GHz}$ [54] showing a quality factor of about 330,000 in a 8-T magnetic field. Further tests are ongoing at LNF with Nb3Sn.

In conclusion, the optimization of the magnetic volume together with the quantumlimited readout and superconductive cavities will allow the LNF haloscope to perform an axion search with a scan speed up to about $20 \mathrm{MHz}$ per day.

Further improvements are expected by the development of single microwave photons detectors under study with the project SIMP [55-58] or the H2020-FET project Super- 
galax [59]. Within the SIMP project, current biased Josephson Junctions (CBJJ) are tested at LNF as switching detector as described in [60]. Recently, a chip fabricated at the CNR-IFN in Rome with Al transmission lines terminated by a single JJ or a DC-SQUID was tested at $10 \mathrm{mK}$ (left panel in Figure 2) to determine the sensitivity to RF photons.

\subsection{From KLASH to FLASH search for Axions with a Large Volume Haloscope}

The KLASH experiment [40] (KLASH, KLoe magnet for Axion SearcH) proposes the realization of a haloscope devoted to the detection for sub- $\mu \mathrm{eV}$ axion by recycling the no-longer used KLOE [61] magnet and the DA $\Phi$ NE cryogenic plant. KLOE is a superconducting solenoid magnet with a very large size bore, able to accommodate a cryrogenic resonant cavity with a diameter of up to $3.7 \mathrm{~m}$.

With the setup described in the CDR [40], it is possible to search for axion scanning in the frequency range of $65-225 \mathrm{MHz}$, corresponding to the well motivated mass region between 0.3 and $1 \mu \mathrm{eV}$.

On Table 1, the KLASH main parameters are shown for the case of the axion's lowest detectable mass. Here, $m_{a}$ is the axion mass, $g_{a \gamma \gamma}^{K S V Z}$ is the axion's coupling parameter of the KSVZ model, $P_{\text {sig }}$ is the signal power, Rate is the expected axion's conversion frequency, $B_{\max }$ is the nominal KLOE magnetic field, $\beta$ is the coupling between cavity and receiver, $\tau$ is the acquisition time, $T_{s y s}$ is the effective noise temperature of the system, and $g_{a \gamma \gamma}$ is the expected axion-photon coupling for the considered $m_{a}$.

Figure 3 (left) shows the expected exclusion limits on $g_{a \gamma \gamma}$ for KLASH in the $0.3-1 \mu \mathrm{eV}$ mass range, compared with the expectations from the KSVZ and DFSZ models. The exclusion limits of other existing experiments are also highlighted.

Table 1. Summary of the KLASH parameterd and discovery potential for KSVZ axions.

\begin{tabular}{cc}
\hline Parameter & Value \\
\hline$m_{a}[\mu \mathrm{eV}]$ & 0.27 \\
$g_{a \gamma \gamma}^{K S V Z}\left[\mathrm{GeV}^{-1}\right]$ & $1.0 \times 10^{-16}$ \\
$P_{\text {sig }}[\mathrm{W}]$ & $1.33 \times 10^{-22}$ \\
Rate $[\mathrm{Hz}]$ & 3050 \\
$B_{\text {max }}[\mathrm{T}]$ & 0.6 \\
$\beta$ & 2 \\
$\tau[\mathrm{min}]$ & 5 \\
$T_{\text {sys }}[\mathrm{K}]$ & 4.9 \\
$g_{a \gamma \gamma} 90 \%$ c.l. $\left[\mathrm{GeV}^{-1}\right]$ & $5.3 \times 10^{-17}$ \\
\hline
\end{tabular}
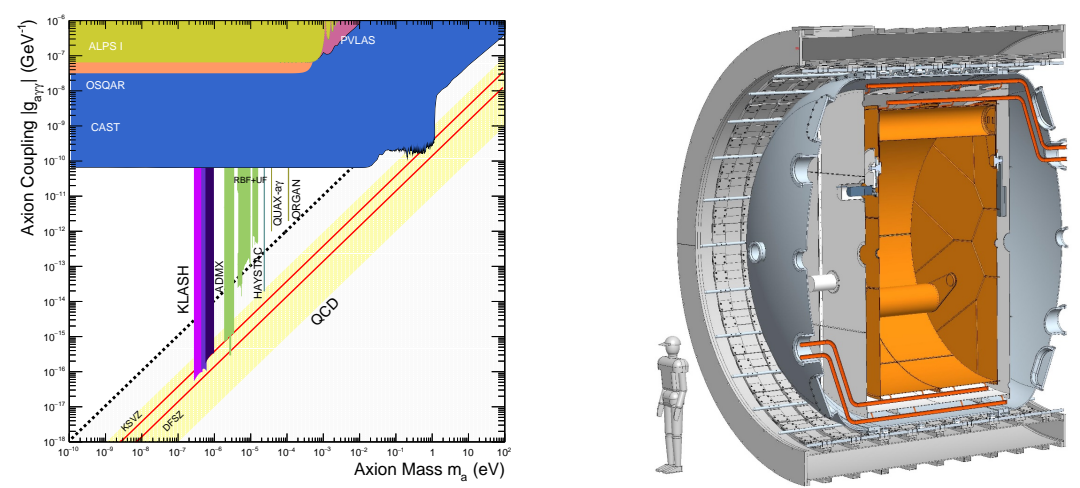

Figure 3. Left: the KLASH discovery potential compared to existing experimental limits. Right: a section view of the KLOE magnet surrounding the cryostat with its radiation shield and the copper cavity. 
Simulations showed that the quality factor $\mathrm{Q}$ for a copper cavity cooled at $4 \mathrm{~K}$ lies in the range of $3 \div 7 \times 10^{5}$ in the considered frequency range. This implies that, in order to complete the frequency scan, 3.5 years of integral data taking should be foreseen.

The cavity signal readout is based on a cryogenic amplification stage (a microstrip SQUID amplifier) followed by a room $\mathrm{T}$ readout chain, which comprises an RF amplifier followed by an intermediate frequency mixer and a near audio frequency mixer stage, the last two arranged in a double-heterodyne receiver.

In the CDR, a preliminary study was carried out about the feasibility and the cost estimate of the realisation and the installation of a $4 \mathrm{~K}$ cryostat containing the copper cavity above mentioned. The cryostat has to be designed to fit and be inserted in KLOE magnet bore (Figure 3 right) and should be equipped with a cryogenic turret, which acts as an interface with the cooling plant. The turret supplies the $4 \mathrm{~K}$ liquid and $70 \mathrm{~K}$ gaseous Helium to the cavity and the radiation shield, respectively.

Recently, INFN designated the KLOE magnet to another experiment (DUNE) [62]. Following this decision, the collaboration is considering the possibility to operate the FINUDA [63] magnet in its place changing the acronym to FLASH (Finuda magnet for Light Axion SearcH).

FINUDA is a superconducting solenoid similar to KLOE, placed in the DA $\Phi N E$ hall at LNF and no longer in use since 2007. The differences respect to KLOE lie in the stronger magnet field (1.1 vs. $0.6 \mathrm{~T}$ ) and a smaller bore size ( 2.7 vs. $4.8 \mathrm{~m}$ in diameter). From the axion sensitivity calculation point of view, these differences roughly compensate; therefore, the expected signal power is very similar for both magnets. The frequency range will be higher than KLOE, due to the smaller dimension of the cavity. Two options are under investigation for the FLASH experimental area: either the present location in the DA $\Phi N E$ hall or in the KLOE hall after the KLOE magnet will be moved.

\section{Dark Photon Search}

The "dark photon" $\left(A^{\prime}\right)$ is the mediator of a new fifth force, introduced in several extensions of the SM, able to connect the dark matter, confined in the dark sector, to the ordinary matter [64]. In this class of models, the fifth force has a U(1) gauge structure and the force carrier has the quantum numbers of a vector particle, just like the SM photon [65]. Due to its electromagnetic-like behavior, the new force, often referred as "portal" interaction, has a very rich phenomenology allowing it to connect any charged particle and photons to the dark photons and potentially to the dark matter.

Several different production mechanisms exist for the $A^{\prime}$, ranging from the analogous of most common standard model electromagnetic interactions, like $\mathrm{A}^{\prime}$-Strahlung $\left(e^{-} N \rightarrow\right.$ $\left.e^{-} N A^{\prime}\right)$ and associated production $\left(e^{+} e^{-} \rightarrow \gamma A^{\prime}\right)$, to meson decays $\left(\pi^{0}, \eta \rightarrow \gamma A^{\prime}\right)$. In all this examples, the dark photon substitutes a SM photon in the EM process of interest. This characteristic of the new particle to mix with the standard model photon, kinetic mixing, allows model builders to exploit the $A^{\prime}$ to explain several different low energy anomalies of the SM.

Among the most popular, and very recently confirmed, is the $(g-2)_{\mu}[66]$. According to [67], the discrepancy in between SM theory and experimental values of $(g-2)_{\mu}$ can be solved by the existence of an $A^{\prime}$ satisfying the following mass to coupling relation:

$$
\epsilon^{2}\left(\frac{100 M e V}{M_{A^{\prime}}}\right)^{2}<1 \times 10^{-3}
$$

which forces a weakly coupled particle able to fix the anomaly to have a mass in the few $\mathrm{MeV}$ scale, exactly in the region where the Frascati facilities are the most effective. As recently suggested by E. Nardi et al. [68], the use of positrons as a beam particle turns on a new production mechanism - the so called "resonant production" $\left(e^{+} e^{-} \rightarrow A^{\prime}\right)$, which will be of crucial importance once a target mass for the candidate is identified. This is the case of the recently discovered ${ }^{8} \mathrm{Be}[69]$ and ${ }^{4} \mathrm{He}$ anomalies, pointing to the existence of a $\sim 17 \mathrm{MeV}$ mass new particle. 


\subsection{The PADME Experiment}

The Positron Annihilation into Dark Matter Experiment (PADME) [70] was developed. PADME is a fixed target experiment installed at BTF beam line 1 (BTF-1) of LNF. Its goal is to search for a massive dark photon $A^{\prime}$ produced in the annihilation of a beam positron with an electron in a thin target:

$$
e^{+} e^{-} \rightarrow \gamma A^{\prime}
$$

The signature of the $A^{\prime}$ production is represented by the missing mass in the reaction (4).

$$
m_{\text {miss }}=\sqrt{\left(P_{e^{+}}+P_{e^{-}}-P_{\gamma}\right)^{2}}
$$

where $P_{i}\left(i=e^{+}, e^{-}, \gamma\right)$ represents the four-momentum of the $i$-particle. Due to the closed kinematics of (4), the existence of an $A^{\prime}$ will appear as a peak on the background distribution of single photon final states, independently of its decay modes. By assuming that the electrons in the target, with mass $m_{e}$, are at rest, and that the positron beam energy is $E_{\text {beam }}$, (5) becomes:

$$
m_{\text {miss }}=\sqrt{2 m_{e}\left[E_{\text {beam }}-E_{\gamma}\left(1+\frac{E_{\text {beam }}}{2 m_{e}} \theta^{2}\right)\right]}
$$

where $E_{\gamma}$ and $\theta$ are the energy and the emission angle, respectively, with respect to the beam direction of the final state photon.

Relation (6) shows that it is fundamental for the experiment to measure, with high efficiency and precision, the 4-momentum of the photons produced at the target.

The decay modes of the $A^{\prime}$ are determined by its mass and by the mass of the particles populating the dark sector. If the mass of the dark photon is lighter than twice the mass of any dark particle $\chi$, it will only decay into SM particles. If $m_{A^{\prime}}<2 m_{e}$ the only accessible process would be three photons final state, via an electron box diagram. Otherwise, the $A^{\prime}$ would decay in leptonic final states. In both these cases, it will be a long lived particle. In the hypothesis that particles in the dark sector are lighter than the dark photon exit, the dominant decay mode will be the invisible channel.

The PADME detector was primarily designed to detect $A^{\prime}$ production. In the first phase of the experiment invisible decay modes of this boson will be explored. In case any hints of a new particle are detected, it is possible to modify the setup to study its decay modes.

\subsection{The PADME Detector}

Figure 4 shows the schematic view of the PADME experimental apparatus.

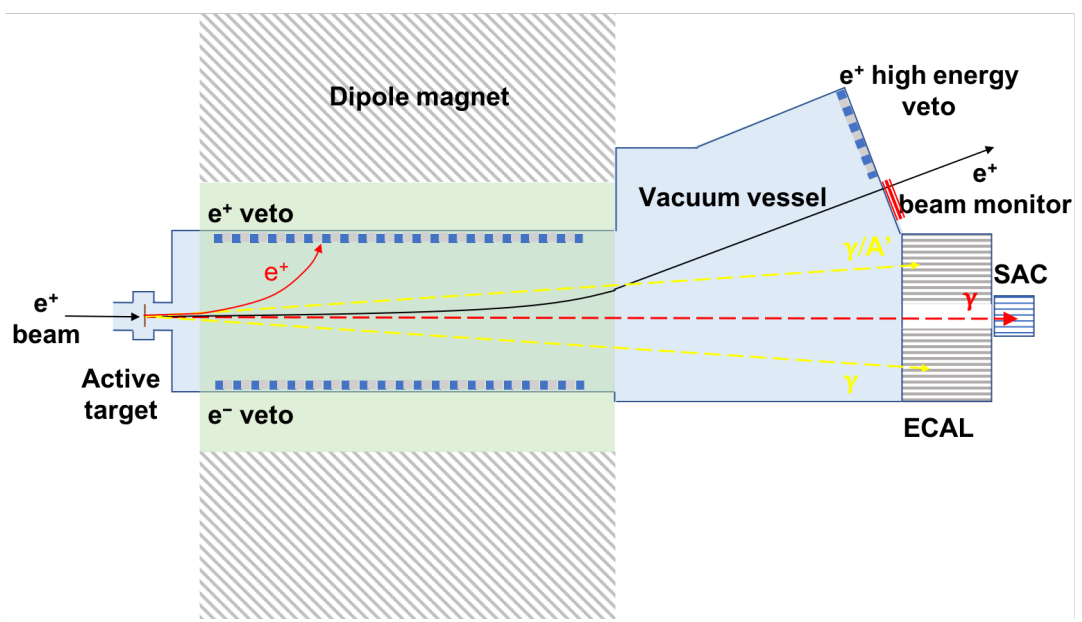

Figure 4. Schematic, top view of the PADME detector. The main components are indicated together with the tracks corresponding to a Bremsstrahlung event (red) and a $\gamma \gamma$ event (yellow) (see text for more details). 
The setup consists of the following components:

- a high intensity and low divergence positron beam, impinging on a thin, active target, capable of monitoring the beam size and intensity (maximum energy $550 \mathrm{MeV}$ );

- a vacuum vessel to avoid spurious particle interactions;

- a dipole magnet to deflect the beam of positrons emerging from the target, with the additional task of enabling the measurement of the momentum of the interacting positrons, thus, allowing the rejection of the Bremsstrahlung background; and

- a finely-segmented, high-resolution e.m. calorimeter, to measure the momentum of the single SM photons (ECal) [71].

Since the processes that mainly take place in the beam-target interaction are Bremsstrahlung and $e^{+} e^{-} \rightarrow \gamma \gamma(\gamma)$, to reject these background events, two extra components are crucial:

- $\quad$ A fast Small Angle Calorimeter (SAC), placed behind the central hole of the ECal. This is used to detect and veto backgrounds photons (mainly from Bremsstrahlung) [72].

- Three stations of plastic scintillator fingers, located inside the vacuum chamber, two within the dipole magnet gap ( $e^{+}$veto and $e^{-}$veto), and the third one on the beam exit (high energy veto), to veto charged particles produced in the interaction [73].

The signals of all PADME sub-detectors are digitized with CAEN V1742 modules [74], based on the DRS4 domino chip able to digitize up to 32 channels, with a maximum selectable sampling rate of $5 \mathrm{GS} / \mathrm{s}$. The main DAQ trigger is provided by the DA $\Phi$ NE LINAC with a rate of $50 \mathrm{~Hz}$, while, for calibration purposes, there are two other triggers signals: a cosmic ray trigger, for ECal calibrations, and a random trigger, to study pedestals and the noise of all detectors.

The PADME detector was built and installed in 2018. It was commissioned on September 2018 and took data until the end of February 2019 (Run1) with a bunch multiplicity of about 20,000-30,000 positrons distributed over $250 \mathrm{nsec}$ long beam bunches. The collected data $\left(\sim 7.5 \times 10^{12}\right.$ POT $)$ were very useful to setup the beam line, to calibrate the detectors, and to establish the running conditions for the real physics run foreseen for 2020. This first data taking was mostly performed with positrons produced on a secondary target installed on the BTF beam-line, and only a small fraction with a primary positron beam produced and accelerated in the LINAC.

This choice, made to have a slightly higher energy giving access to a wider region of $A^{\prime}$ masses, turned out to be not optimal for the experiment. In fact, the data analysis clearly showed a relevant beam-induced background related to the beam-line structure. Therefore, a new technical run (summer 2020) was performed with primary positrons and a redesigned beam line. At the end of this running period, the background conditions were strongly reduced, and a second data taking (Run2) was performed. During this period $\approx 5 \times 10^{12}$ POT were collected.

\subsection{Dark Sector Search with PADME}

As already pointed out, PADME is looking for dark sectors signals selecting mono photon final states resulting from the positrons annihilation on the electrons of the target. Referring to Figure 4, a dark sector candidate is represented in the PADME setup by a single cluster detected by the ECAL and nothing else in the other detectors.

These kinds of events have to be isolated from the more abundant final states with one or more photons, including positron annihilation and positron Bremsstrahlung events. Table 2 reports the cross-sections for these different SM reactions evaluated for a positron energy of $550 \mathrm{MeV}$. 
Table 2. Main interactions occurring in $e^{+} e^{-}$collisions and corresponding values of cross-sections evaluated for a positron energy of $550 \mathrm{MeV}$.

\begin{tabular}{cc}
\hline Process & $\sigma[\mathrm{mb}]$ \\
\hline$e^{+} e^{-} \rightarrow \gamma \gamma$ & 1.55 \\
$e^{+} N \rightarrow e^{+} N \gamma$ & 4000 \\
$e^{+} e^{-} \rightarrow \gamma \gamma \gamma$ & 0.16 \\
\hline
\end{tabular}

The conditions to select mono-photon final states include no extra photons with energy above $50 \mathrm{MeV}$ in the calorimeters, $\mathrm{ECal}$ and $\mathrm{SAC}$, in within a $\pm 2 \mathrm{~ns}$ time window. To reject residual Bremsstrahlung background, no hits in the vetoes in time coincidence ( $\pm 2 \mathrm{~ns})$ with the single ECAL cluster and with a positrons energy compatible with $E_{\text {beam }}-E_{\text {Cluster }}$ are allowed. The lower limit on the mixing constant $\varepsilon$ measurable in PADME is about $10^{-3}$ and is related to the maximum number of positrons per bunch, compatible with a pile-up manageable by the detector.

The limit on the mass of the $A^{\prime}$ is related to the beam energy; for a beam energy of $550 \mathrm{MeV}$, an upper value of $23.7 \mathrm{MeV} / c^{2}$ for the mass of the dark photon can be obtained. The excluded region in the parameter space $\left(m_{A^{\prime}}, \varepsilon^{2}\right)$ for invisible decay of the dark photon, obtained for different values of the integrated luminosity, is shown in Figure 5.

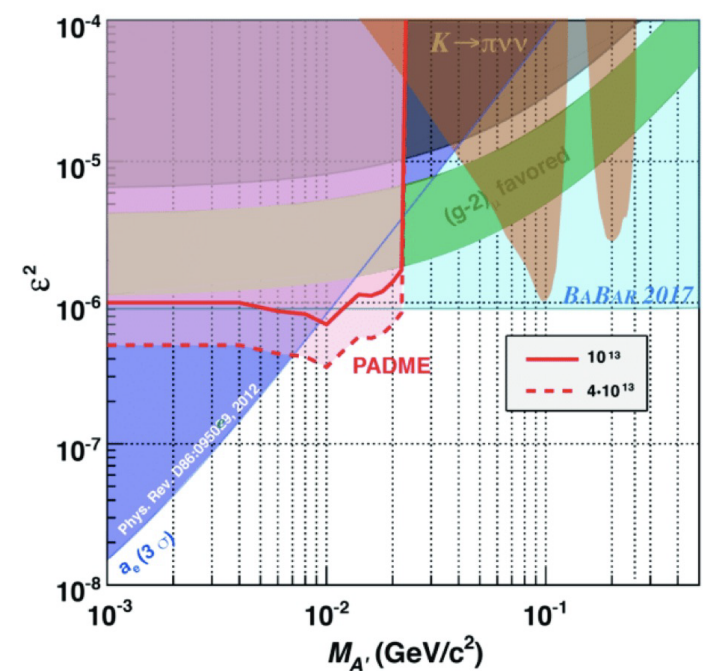

Figure 5. PADME exclusion region of the parameter space $\left(\varepsilon^{2}, M_{A^{\prime}}\right)$ for different values of the integrated luminosity.

More recently the possibility of extended dark sector searches has been explored, including in the PADME physics program the study of ALPs coupled to electrons and photons [75] and dark Higgs [76].

\subsection{Improvements of the Positron Beam}

The main limitation to the statistics of the PADME experiment (or any future modified version of it) comes from the need of keeping the pile-up and the over-veto probabilities under control, both in the calorimeters and in the scintillating bars for the charged particle detection. For this reason, the positron beam has to be diluted in time as much as possible: in the 2018-2020 data, with the PADME setup installed on the BTF-1 line, the positron beam intensity was limited depending on the beam pulse length $\Delta t$ with the following rule-of-thumb:

$$
n_{e^{+}} \sim 10^{2} \times \Delta t .
$$

The pulse length ranged between 150 up to $320 \mathrm{~ns}$, with slightly different maximum beam energies, due to the two different positron production methods used and the modi- 
fications to the accelerating RF needed for extending the beam pulse duration [77]. The "primary" positron beam is delivered by the DA $\Phi$ NE LINAC striking electrons, emitted by the thermo-ionic gun and accelerated to an intermediate energy by the first sections, onto a "positron converter" target; the produced positrons are, then, brought to the maximum energy by the following accelerating sections.

In order to produce a lower intensity but higher energy positron beam, all LINAC sections are used instead for accelerating primary electrons and strike them at the maximum energy onto the attenuating target of the BTF beam-line. Higher energy "secondary" positrons produced in the resulting shower are then momentum-selected by the BTF dipole and collimators. The main parameters of the resulting positron beam with these two different configurations are listed in Table 3.

Table 3. Main positron beam parameters from DAФNE LINAC in the 2018-2020 PADME data.

\begin{tabular}{ccc}
\hline & Primary $e^{+}$ & Secondary $e^{+}$ \\
\hline Pulse length & \multicolumn{2}{c}{$150-320 \mathrm{~ns}$} \\
\hline Maximum energy & $490 \mathrm{MeV}$ & $545 \mathrm{MeV}$ \\
\hline Maximum intensity & $6 \times 10^{10}$ & $10^{5}$ \\
\hline
\end{tabular}

The different scenarios for performing dark sector searches using the PADME setup or a PADME-like experiment and the existing Frascati accelerator complex can be grouped in the following five categories:

1. Further extending the primary positron beam accelerated by the LINAC: due to the sharp shape of the compressed RF pulse needed for reaching higher energies, this is generally achieved at the cost of a reduced maximum energy. A beam length in the $\mu$ s range can be achieved, having, as a reference, minimum energy the kinematic threshold for the production of the hypothetical $17 \mathrm{MeV} / \mathrm{c}^{2}$ boson postulated for accounting the anomalies in the ${ }^{8} \mathrm{Be}$ and ${ }^{4} \mathrm{He}$ IPC transitions, of $\approx 300 \mathrm{MeV}$ [78].

2. Much longer positron beams, in the ms range, with the same particle density can be achieved by using one of the DA $\Phi N E$ main rings, or in the case of the smaller accumulator ring, as an extender of the LINAC beam pulses (POSEYDON project) [79]. The standard technique is exciting a betatron tune resonance (typically close to a third of integer) to increase the oscillations of the circulating positrons and drive them toward an extraction septum.

3. The resonant extraction can be improved by using the deflection of positrons due to coherent processes in bent crystal, like the channeling, volume reflection or mirroring effects (SHERPA project) [80].

4. A possible continuous beam extraction can be obtained profiting of the transverse offset of particles due to an energy loss mechanism: positrons can be scattered by intra-beam elastic interactions (the so-called Touschek effect), or by a thin target (wire) intercepting the beam periphery, or by the photons of a suitable laser beam (inverse Compton effect). Eventually, those stray particles will be further deflected by means of conventional septum magnet.

5. The not-attenuated LINAC pulse, extended to $\approx 300 \mathrm{~ns}$, produces a high intensity electron (or positron) beam, which can be used for performing a thick target experiment in the area of the existing DA $\Phi$ NE LINAC dump.

\section{Conclusions}

The Frascati National Laboratory is the largest and the first built among the INFN Laboratories. Since its foundation, it has been devoted to two main activities: the development, construction, and operation of accelerators; and the design and construction of the forefront of detectors for particle, nuclear, and astro-particle experiments. 
At present, in operation at LNF are two accelerator facilities: the DAФNE complex that is completing its scientific program with measurements of low-energy QCD and provides synchrotron radiation or test-beams to external users, and the SPARC_LAB where a bright photo-injector, combined with the FLAME laser, constitute a source of high-quality radiations and the environment to develop new techniques for compact particle accelerators. Due to the research activities ongoing at the SPARC_LAB, LNF has identified its future in the EuPRAXIA project and has put forward its candidacy to host one of the major pillars of this International infrastructure.

This program is moving away from fundamental research in the scientific interests of the laboratory. Therefore, in order to maintain the capability to contribute to basic science, a widening of the traditional scientific interests of the laboratory is taking place by exploiting the presently available infrastructures. These can be used to contribute to the world effort to identify dark matter signals. Two complementary research programs are being pursued currently at LNF: the search for QCD axions with resonant detectors and the identification of dark photon signals at accelerators. Both activities are mature and will deliver results in the next decade.

Author Contributions: Conceptualization, P.G.; Axions sessions, C.G. and C.L.; Dark Photon sessions, P.G. and M.R.; Positron beam, P.V. All authors have read and agreed to the published version of the manuscript.

Funding: This research received no external funding.

Institutional Review Board Statement: Not applicable.

Informed Consent Statement: Not applicable.

Data Availability Statement: Not applicable.

Conflicts of Interest: The authors declare no conflicts of interest.

\section{References}

1. Alesini, D.; Anania, M.P.; Artioli, M.; Bacci, A.; Bartocci, S.; Bedogni, R.; Bellaveglia, M.; Biagioni, A.; Bisesto, F.; Brandi, F.; et al. EuPRAXIA@SPARC_LAB Conceptual Design Report. 2018. Available online: http://www.lnf.infn.it/sis/preprint/pdf/getfile. php?filename=INFN-18-03-LNF.pdf (accessed on 7 July 2021)

2. Assmann, R.W.; Weikum, M.K.; Akhter, T.; Alesini, D.; Alexandrova, A.S.; Anania, M.P.; Andreev, N.E.; Andriyash, I.; Artioli, M.; Aschikhin, A.; et al. EuPRAXIA Conceptual Design Report. Eur. Phys. J. ST 2020, 229, 3675-4284; Erratum in 2020, $229,11-31$. [CrossRef]

3. Bernardini, C.; Bizzarri, U.; Corazza, G.F.; Ghigo, G.; Querzoli, R.; Touschek, B. A 250-Mev Electron-Positron Storage Ring: The "A D A". In Proceedings of the 3rd International Conference on High-Energy Accelerators, HEACC 1961. Upton, NY, USA, 6-12 September 1961; pp. 256-261.

4. Amman, F.; Andreani, R.; Bassetti, M.; Bernardini, M.; Cattoni, A. ; Chimenti, V.; Corazza, G.F.; Dehne, H.C.; Fabiani, D.; Massarotti, A.; et al. Single and two beam operation in adone. In Proceedings of the 7th International Conference on High-Energy Accelerators, HEACC 1969. Yerevan, USSR, 27 August 27-2 September 1969; pp. 9-18.

5. Zobov, M. DAFNE status and upgrade plans. Phys. Part. Nucl. Lett. 2008, 5, 560-565. [CrossRef]

6. Bloise, C. Hadron physics with KLOE data at the $\phi$-factory. AIP Conf. Proc. 2020, 2249, 020004. [CrossRef]

7. Anastasi, A.; Babusci, D.; Bencivenni, G.; Berlowski, M.; Bloise, C.; Bossi, F.; Branchini, P.; Budano, A.; Balkeståhl, L.C.; Cao, B.; et al. Measurement of the running of the fine structure constant below $1 \mathrm{GeV}$ with the KLOE Detector. Phys. Lett. B 2017, 767, 485-492. [CrossRef]

8. Zmeskal, J.; Beer, G.; Bragadirean, A.M.; Cargnelli, M.; Curceanu, C.; Egger, J.P.; Fuhrmann, H.; Guaraldo, C.; Iliescu, M.; Ishiwatari, T.; et al. The DEAR experiment: First results on kaonic hydrogen. Nucl. Phys. A 2005, 754, 369-374. [CrossRef]

9. Bazzi, M.; Beer, G.; Bombelli, L.; Bragadireanu, A.M.; Cargnelli, M.; Corradi, G.; Curceanu, C.; d’Uffizi, A.; Fiorini, C.; Frizzi, T.; et al. First measurement of kaonic helium-3 X-rays. Phys. Lett. B 2011, 697, 199-202. [CrossRef]

10. Agnello, M.; Benussi, L.; Bertani, M.; Bhang, H.C.; Bonomi, G.; Botta, E.; Bregant, M.; Bressani, T.; Bufalino, S.; Busso, L.; et al. Hypernuclear spectroscopy with $\mathrm{K}^{-}$at rest on ${ }^{7} \mathrm{Li},{ }^{9} \mathrm{Be},{ }^{13} \mathrm{C}$ and ${ }^{16} \mathrm{O}$. Phys. Lett. B 2011, 698, 219-225. [CrossRef]

11. Valente, P.; Belli, M.; Bolli, B.; Buonomo, B.; Cantarella, S.; Ceccarelli, R.; Cecchinelli, A.; Cerafogli, O.; Clementi, R.; Di Giulio, C.; et al. Linear Accelerator Test Facility at LNF: Conceptual Design Report. arXiv 2016, arXiv:1603.05651.

12. Ferrario, M.; Alesini, D.; Anania, M.; Bacci, A.; Bellaveglia, M.; Bogdanov, O.; Boni, R.; Castellano, M.; Chiadroni, E.; Cianchi, A.; et al. SPARC_LAB present and future. Nucl. Instrum. Meth. B 2013, 309, 183-188. [CrossRef] 
13. Bahcall, N.A.; Ostriker, J.P.; Perlmutter, S.; Steinhardt, P.J. The Cosmic triangle: Assessing the state of the universe. Science 1999, 284, 1481-1488. [CrossRef]

14. Spergel, D.N.; Steinhardt, P.J. Observational evidence for selfinteracting cold dark matter. Phys. Rev. Lett. 2000, 84, 3760-3763. [CrossRef]

15. Battaglieri, M.; Belloni, A.; Chou, A.; Cushman, P.; Echenard, B.; Essig, R.; Estrada, J.; Feng, J.L.; Flaugher, B.; Fox, P.J.; et al. US Cosmic Visions: New Ideas in Dark Matter 2017: Community Report. arXiv 2017, arXiv:1707.04591.

16. Jungman, G.; Kamionkowski, M.; Griest, K. Supersymmetric dark matter. Phys. Rep. 1996, 267, 195-373. [CrossRef]

17. Preskill, J.; Wise, M.B.; Wilczek, F. Cosmology of the Invisible Axion. Phys. Lett. B 1983, 120, 127-132. [CrossRef]

18. Peccei, R.D.; Quinn, H.R. Constraints Imposed by CP Conservation in the Presence of Instantons. Phys. Rev. D 1977, 16, 1791-1797. [CrossRef]

19. Arcadi, G.; Dutra, M.; Ghosh, P.; Lindner, M.; Mambrini, Y.; Pierre, M.; Profumo, S.; Queiroz, F.S. The waning of the WIMP? A review of models, searches, and constraints. Eur. Phys. J. C 2018, 78, 203. [CrossRef]

20. Essig, R.; Jaros, J.A.; Wester, W. Dark Sectors and New, Light, Weakly-Coupled Particles. arXiv 2013, arXiv:1311.0029.

21. Alexander, J.; Battaglieri, M.; Echenard, B.; Essig, R.; Graham, M.; Izaguirre, E.; Jaros, J.; Krnjaic, G.; Mardon, J.; Morrissey, D.; et al. Dark Sectors 2016 Workshop: Community Report. arXiv 2016, arXiv:1608.08632.

22. Weinberg, S. A New Light Boson? Phys. Rev. Lett. 1978, 40, 223-226. [CrossRef]

23. Wilczek, F. Problem of Strong P and T Invariance in the Presence of Instantons. Phys. Rev. Lett. 1978, 40, 279-282. [CrossRef]

24. Peccei, R.D.; Quinn, H.R. CP Conservation in the Presence of Pseudoparticles. Phys. Rev. Lett. 1977, 38, 1440-1443. [CrossRef]

25. Abbott, L.; Sikivie, P. A cosmological bound on the invisible axion. Phys. Lett. B 1983, 120, 133-136. [CrossRef]

26. Dine, M.; Fischler, W. The not-so-harmless axion. Phys. Lett. B 1983, 120, 137-141. [CrossRef]

27. Ipser, J.; Sikivie, P. Can galactic halos be made of Axions? Phys. Rev. Lett. 1983, 50, 12. [CrossRef]

28. Sikivie, P. Experimental Tests of the "Invisible" Axion. Phys. Rev. Lett. 1983, 51, 1415-1417. [CrossRef]

29. Sikivie, P. Detection rates for "invisible"-axion searches. Phys. Rev. D 1985, 32, 2988-2991. [CrossRef] [PubMed]

30. Alesini, D.; Braggio, C.; Carugno, G.; Crescini, N.; D’Agostino, D.; Di Gioacchino, D.; Di Vora, R.; Falferi, P.; Gambardella, U.; Gatti, C.; et al. Search for Invisible Axion Dark Matter of mass $m_{a}=43 \mu \mathrm{eV}$ with the QUAX-a $\gamma$ Experiment. arXiv 2020, arXiv:2012.09498.

31. Du, N.; Force, N.; Khatiwada, R.; Lentz, E.; Ottens, R.; Rosenberg, L.J.; Rybka, G.; Carosi, G.; Woollett, N.; Bowring, D.; et al. Search for invisible axion dark matter with the axion dark matter experiment. Phys. Rev. Lett. 2018, 120, 151301. [CrossRef]

32. Braine, T.; Cervantes, R.; Crisosto, N.; Du, N.; Kimes, S.; Rosenberg, L.J.; Rybka, G.; Yang, J.; Bowring, D.; Chou, A.S.; et al. Extended search for the invisible axion with the axion dark matter experiment. Phys. Rev. Lett. 2020, 124, 101303. [CrossRef]

33. Zhong, L.; Al Kenany, S.; Backes, K.M.; Brubaker, B.M.; Cahn, S.B.; Carosi, G.; Gurevich, Y.V.; Kindel, W.F.; Lamoreaux, S.K.; Lehnert, K.W.; et al. Results from phase 1 of the HAYSTAC microwave cavity axion experiment. Phys. Rev. D 2018, $97,092001$. [CrossRef]

34. Backes, K.M.; Palken, D.A.; Al Kenany, S.; Brubaker, B.M.; Cahn, S.B.; Droster, A.; Hilton, G.C.; Ghosh, S.; Jackson, H.; Lamoreaux, S.K.; et al. A quantum-enhanced search for dark matter axions. Nature 2021, 590, 238. [CrossRef]

35. McAllister, B.T.; Flower, G.; Ivanov, E.N.; Goryachev, M.; Bourhill, J.; Tobar, M.E. The ORGAN experiment: An axion haloscope above 15 GHz. Phys. Dark Universe 2017, 18, 67-72. [CrossRef]

36. Jeong, J.; Bae, S.; Semertzidis, Y.K.; Youn, S.; Kim, J.; Seong, T.; Kim, J.E. Search for invisible axion dark matter with a multiple-cell haloscope. Phys. Rev. Lett. 2020, 125, 221302. [CrossRef]

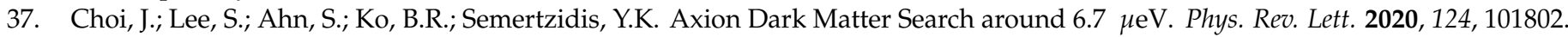
[CrossRef]

38. Melcón, A.Á.; Cuendis, S.A.; Cogollos, C.; Díaz-Morcillo, A.; Döbrich, B.; Gallego, J.D.; Gimeno, B.; Irastorza, I.G.; LozanoGuerrero, A.J.; Malbrunot, C.; et al. Axion searches with microwave filters: The RADES project. J. Cosmol. Astropart. Phys. 2018, 2018, 040. [CrossRef]

39. Crescini, N.; Alesini, D.; Braggio, C.; Carugno, G.; D’Agostino, D.; Di Gioacchino, D.; Falferi, P.; Gambardella, U.; Gatti, C.; Iannone, G.; et al. Axion Search with a Quantum-Limited Ferromagnetic Haloscope. Phys. Rev. Lett. 2020, 124, 171801. [CrossRef]

40. Alesini, D.; Babusci, D.; Björkeroth, F.; Bossi, F.; Ciambrone, P.; Monache, G.D.; Di Gioacchino, D.; Falferi, P.; Gallo, A.; Gatti, C.; et al. KLASH Conceptual Design Report. arXiv 2019, arXiv:1911.02427.

41. Caldwell, A.; Dvali, G.; Majorovits, B.; Millar, A.; Raffelt, G.; Redondo, J.; Reimann, O.; Simon, F.; Steffen, F.; MADMAX Working Group. Dielectric Haloscopes: A New Way to Detect Axion Dark Matter. Phys. Rev. Lett. 2017, 118, 091801. [CrossRef]

42. BRASS: Broadband Radiometric Axion SearcheS. Available online: http://wwwiexp.desy.de/groups/astroparticle/brass/ brassweb.htm (accessed on 7 July 2021).

43. Ouellet, J.L.; Salemi, C.P.; Foster, J.W.; Henning, R.; Bogorad, Z.; Conrad, J.M.; Formaggio, J.A.; Kahn, Y.; Minervini, J.; Radovinsky, A.; et al. First Results from ABRACADABRA-10 cm: A Search for Sub- $\mu$ eV Axion Dark Matter. Phys. Rev. Lett. 2019, 122, 121802. [CrossRef]

44. Aybas, D.; Adam, J.; Blumenthal, E.; Gramolin, A.V.; Johnson, D.; Kleyheeg, A.; Afach, S.; Blanchard, J.W.; Centers, G.P.; Garcon, A.; et al. Search for Axionlike Dark Matter Using Solid-State Nuclear Magnetic Resonance. Phys. Rev. Lett. 2021, 126, 141802. [CrossRef] 
45. Barbieri, R.; Braggio, C.; Carugno, G.; Gallo, C.; Lombardi, A.; Ortolan, A.; Pengo, R.; Ruoso, G.; Speake, C. Searching for galactic axions through magnetized media: The QUAX proposal. Phys. Dark Universe 2017, 15, 135-141. [CrossRef]

46. Crescini, N.; Alesini, D.; Braggio, C.; Carugno, G.; Di Gioacchino, D.; Gallo, C.S.; Gambardella, U.; Gatti, C.; Iannone, G.; Lamanna, G.; et al. Operation of a ferromagnetic axion haloscope at $m_{a}=58 \mu \mathrm{eV}$. Eur. Phys. J. C 2018, 78. [CrossRef]

47. Alesini, D.; Braggio, C.; Carugno, G.; Crescini, N.; D’Agostino, D.; Di Gioacchino, D.; Di Vora, R.; Falferi, P.; Gallo, S.; Gambardella, U.; et al. Galactic axions search with a superconducting resonant cavity. Phys. Rev. D 2019, 99, 101101. [CrossRef]

48. Di Gioacchino, D.; Gatti, C.; Alesini, D.; Ligi, C.; Tocci, S.; Rettaroli, A.; Carugno, G.; Crescini, N.; Ruoso, G.; Braggio, C.; et al. Microwave Losses in a DC Magnetic Field in Superconducting Cavities for Axion Studies. IEEE Trans. Appl. Supercon. 2019, 29, 1-5. [CrossRef]

49. Alesini, D.; Braggio, C.; Carugno, G.; Crescini, N.; D’Agostino, D.; Di Gioacchino, D.; Di Vora, R.; Falferi, P.; Gambardella, U.; Gatti, C.; et al. High quality factor photonic cavity for dark matter axion searches. Rev. Sci. Instrum. 2020, 91, 094701. [CrossRef] [PubMed]

50. Alesini, D.; Braggio, C.; Carugno, G.; Crescini, N.; D’ Agostino, D.; Di Gioacchino, D.; Di Vora, R.; Falferi, P.; Gambardella, U.; Gatti, C.; et al. Realization of a high quality factor resonator with hollow dielectric cylinders for axion searches. Nucl. Instrum. Methods A 2021, 985, 164641. [CrossRef]

51. COLD Lab. Available online: http:/ / coldlab.lnf.infn.it (accessed on 7 July 2021)

52. Zorin, A.B. Josephson Traveling-Wave Parametric Amplifier with Three-Wave Mixing. Phys. Rev. Appl. 2016, 6, 034006. [CrossRef]

53. Heinsoo, J.; Andersen, C.K.; Remm, A.; Krinner, S.; Walter, T.; Salathé, Y.; Gasparinetti, S.; Besse, J.C.; Potočnik, A.; Wallraff, A.; et al. Rapid High-fidelity Multiplexed Readout of Superconducting Qubits. Phys. Rev. Appl. 2018, 10, 034040. [CrossRef]

54. Ahn, D.; Kwon, O.; Chung, W.; Jang, W.; Lee, D.; Lee, J.; Youn, S.W.; Youm, D.; Semertzidis, Y.K. Superconducting cavity in a high magnetic field. arXiv 2020, arXiv:2002.08769.

55. Alesini, D.; Babusci, D.; Barone, C.; Buonomo, B.; Beretta, M.M.; Bianchini, L.; Castellano, G.; Chiarello, F.; Gioacchino, D.D.; Falferi, P.; et al. Development of a Josephson junction based single photon microwave detector for axion detection experiments. J. Phys. Conf. Ser. 2020, 1559, 012020. [CrossRef]

56. Alesini, D.; Babusci, D.; Barone, C.; Buonomo, B.; Beretta, M.M.; Bianchini, L.; Castellano, G.; Chiarello, F.; Di Gioacchino, D.; Falferi, P.; et al. Status of the SIMP Project: Toward the Single Microwave Photon Detection. J. Low Temp. Phys. 2020, 199, 348-354. [CrossRef]

57. Piedjou Komnang, A.S.; Guarcello, C.; Barone, C.; Gatti, C.; Pagano, S.; Pierro, V.; Rettaroli, A.; Filatrella, G. Analysis of Josephson junctions switching time distributions for the detection of single microwave photons. Chaos Solitons Fractals 2021, 142, 110496. [CrossRef]

58. Paolucci, F.; Buccheri, V.; Germanese, G.; Ligato, N.; Paoletti, R.; Signorelli, G.; Bitossi, M.; Spagnolo, P.; Falferi, P.; Rajteri, M.; et al. Development of highly sensitive nanoscale transition edge sensors for gigahertz astronomy and dark matter search. J. Appl. Phys. 2020, 128, 194502. [CrossRef]

59. SUPERGALAX H2020-FETOPEN-2018-2019-2020-01. Available online: https:/ / supergalax.eu (accessed on 7 July 2021)

60. Kuzmin, L.S.; Sobolev, A.S.; Gatti, C.; Di Gioacchino, D.; Crescini, N.; Gordeeva, A.; Il'ichev, E. Single Photon Counter based on a Josephson Junction at $14 \mathrm{GHz}$ for searching Galactic Axions. IEEE Trans. Appl. Supercond. 2018, 28, 2400505. [CrossRef]

61. Franzini, P.; Moulson, M. The Physics of DAФNE and KLOE. Annu. Rev. Nucl. Part. Sci. 2006, 56, 207-251. [CrossRef]

62. Abi, B.; Acciarri, R.; Acero, M.A.; Adamov, G.; Adams, D.; Adinolfi, M.; Ahmad, Z.; Ahmed, J.; Alion, T.; Monsalve, S.A.; et al. Experiment Simulation Configurations Approximating DUNE TDR. arXiv 2021, arXiv:2103.04797.

63. Bertani, M.; Cecchetti, A.; Dulach, B.; Fabbri, F.L.; Giardoni, M.; Lanaro, A.; Lucherini, V.; Bressani, T.; Feliciello, A.; Filippi, A.; et al. The FINUDA superconducting magnet at DAFNE. Nucl. Phys. B Proc. Suppl. 1999, 78, 553-558. [CrossRef]

64. Berezhiani, Z.G.; Dolgov, A.D.; Mohapatra, R.N. Asymmetric inflationary reheating and the nature of mirror universe. Phys. Lett. B 1996, 375, 26-36. [CrossRef]

65. Holdom, B. Searching for $\epsilon$ Charges and a New U(1). Phys. Lett. B 1986, 178, 65-70. [CrossRef]

66. Lee, H.S. Muon g-2 anomaly and dark leptonic gauge boson. Phys. Rev. D 2014, 90, 091702. [CrossRef]

67. Pospelov, M. Secluded U(1) below the weak scale. Phys. Rev. D 2009, 80, 095002. [CrossRef]

68. Nardi, E.; Carvajal, C.D.R.; Ghoshal, A.; Meloni, D.; Raggi, M. Resonant production of dark photons in positron beam dump experiments. Phys. Rev. D 2018, 97, 095004. [CrossRef]

69. Krasznahorkay, A.J.; Csatlós, M.; Csige, L.; Gácsi, Z.; Gulyás, J.; Hunyadi, M.; Kuti, I.; Nyakó; BM; Stuhl, L.; Timár, J.; et al. Observation of Anomalous Internal Pair Creation in Be8: A Possible Indication of a Light, Neutral Boson. Phys. Rev. Lett. 2016, 116, 042501. [CrossRef] [PubMed]

70. Raggi, M.; Kozhuharov, V. Proposal to Search for a Dark Photon in Positron on Target Collisions at DAథNE Linac. Adv. High Energy Phys. 2014, 2014, 959802. [CrossRef]

71. Albicocco, P.; Alexander, J.; Bossi, F.; Branchini, P.; Buonomo, B.; Capoccia, C.; Capitolo, E.; Chiodini, G.; Caricato, A.P.; de Sangro, R.; et al. Characterisation and performance of the PADME electromagnetic calorimeter. JINST 2020, 15, T10003. [CrossRef] 
72. Frankenthal, A.; Alexander, J.; Buonomo, B.; Capitolo, E.; Capoccia, C.; Cesarotti, C.; De Sangro, R.; Di Giulio, C.; Ferrarotto, F.; Foggetta, L.; et al. Characterization and performance of PADME's Cherenkov-based small-angle calorimeter. Nucl. Instrum. Meth. A 2019, 919, 89-97. [CrossRef]

73. Oliva, F. The charged particle veto system of the PADME experiment. Nucl. Instrum. Meth. A 2019, 936, 259-260. [CrossRef]

74. Available online: http://www.caen.it (accessed on 7 July 2021).

75. Darmé, L.; Giacchino, F.; Nardi, E.; Raggi, M. Invisible decays of axion-like particles: Constraints and prospects. arXiv 2020 arXiv:hep-ph/2012.07894.

76. Ciafaloni, P.; Martelli, G.; Raggi, M. Searching for dark sectors in multi lepton final states in $\mathrm{e}^{+} \mathrm{e}^{-}$collisions. JHEP 2021, $04,163$. [CrossRef]

77. Valente, P.; Belli, M.; Buonomo, B.; Ceccarelli, R.; Cecchinelli, A.; Clementi, R.; Di Giulio, C.; Foggetta, L.G.; Piermarini, G.; Rossi, L.A.; et al. Long beam pulses with SLED compression in DAФNE LINAC. J. Phys. Conf. Ser. 2017, 874, 012017. [CrossRef]

78. Valente, P. Ideas for extending the Frascati LINAC positron beam pulses for the resonant search of a X(17 MeV) boson. arXiv 2020, arXiv:2001.10258.

79. Valente, P. POSEYDON-Converting the DA $\Phi$ NE Collider into a double Positron Facility: A High Duty-Cycle pulse stretcher and a storage ring. arXiv 2017, arXiv:1711.06877.

80. Garattini, M.; Gianotti, P.; Guiducci, S.; Liedl, A.; Vaccarezza, C. The SHERPA Experiment. LNF-Activity Report-2020. 2021. Available online: http:/ / www.lnf.infn.it/rapatt/2020/sherpa.pdf (accessed on 7 July 2021) 